\title{
Non-linear Optical Materials at Nanoscale: Synthesis of Second Harmonic Active Lithium Niobate Nanocrystals through Solution-Phase Methods
}

\author{
Rana Faryad Ali, Byron Gates \\ Department of Chemistry, Simon Fraser University, Canada \\ ranaa@sfu.ca,bgates@sfu.ca.
}

\section{Extended Abstract}

We advanced the development of solution-phase approaches for the preparation of lithium niobate (LiNbO3) nanoparticles with an average, tunable size from 7 to $100 \mathrm{~nm}$. This solution-phase process results in the formation of crystalline, uniform nanoparticles of $\mathrm{LiNbO} 3$ at a reaction temperature of $220{ }^{\circ} \mathrm{C}$ with an optimal reaction time of as short as $30 \mathrm{~h}$. Advantages of these methods include the preparation of single-crystalline LiNbO3 nanoparticles without the need for further heat treatment or without the need for using an inert reaction atmosphere. The growth of these nanoparticles began with a controlled agglomeration of nuclei formed during a solvolysis step. The reactions subsequently underwent the processes of condensation, aggregation, and Ostwald ripening, which remained the dominant process during further growth of the nanoparticles. These processes did produce single-crystalline nanoparticles of LiNbO3, suggesting an oriented attachment process. Average dimensions of the nanoparticles were tuned from 7 to $\sim 100 \mathrm{~nm}$ by either increasing the reaction time or changing the concentration of the lithium salts used in the solvothermal process. The nanoparticles were also confirmed to be optically active for second harmonic generation (SHG). These particles could enable further development of SHG based microscopy techniques. 